\title{
Navigation in multi-robot systems based on the behavioural fuzzy controller
}

\author{
Teggar Hamza and Meftah Boudjelal \\ University of Mascara, Dept. of Computer Sciences, 29000, Mascara, Algeria
}

Received: 13 June 2021, Accepted: 16 June 2021

Published online: 12 July 2021.

\begin{abstract}
Controlling a highly dynamic multi-robot system (DMRS) is a challenge scientific and technological in constant expansion. Our goal in this article is to control the DMRS and used the local interactions between the robot and its environment to produce a form of advanced intelligence in the selection of behaviours. For this, we have developed a reactive controller based on fuzzy behaviours using the concept of fuzzy logic. This controller allows mobile robots to move in an unknown environment and, at the same time, to resolve the navigation conflicts. The simulation results obtained on the pioneer P3-DX robot clearly show the effectiveness of the proposed fuzzy controller.
\end{abstract}

Keywords: Multi-robot systems, fuzzy logic, mobile robots, behavioural control architecture.

\section{Introduction}

Controlling a dynamic multi-robot system (DMRS) inevitably involves controlling the actions generated by each robot [1]. In the literature of collective robotics, several approaches have been proposed to control SMRS, such as centralized [2]. and distributed approaches [3]. This article focuses on navigation in DMRS using a distributed control approach. In this context, the navigation methods allow the robot to move from a start point to target point while avoiding obstacles. These methods must therefore take into account the following problems: Fixed Obstacle Avoidance Problem (FOAP); Mobile Obstacle Avoidance Problem (MOAP); and Robots Collision Avoidance Problem (RCAP).

In the FOA Problem, the environment is considered static and contains a single robot [4]. In the MOA Problem, the environment contains dynamic obstacles and several robots [5,7]. Finally, in the RCA Problem, robots could communicate with each other to find an arrangement to avoid collisions between them [8].

In this paper, the FOAP and MOAP will be considered. The main goal of this study is the design of a control architecture based on fuzzy behavioural rules, which exhibit both individual characteristics adapted to dynamic multi-robot systems and collective characteristics that promote a common goal [6]. More specifically, we propose a new process for the selection of navigation behaviours to solve the FOAP and MOAP. The proposed method allows the coordination of elementary primitives (called behaviours) in a flexible way. At the scale of the group of robots, the method also allows to achieve coordination between the robots and promoting the target tracking to solve MOAP.

The remainder of this paper is organized as follows. In the next section, the kinematic model of the robot is presented. The fuzzy controller architecture proposed for navigation will be detailed in the section III. The results of the simulation on the Pioneer 3P-DX robot will be presented in section IV. Finally, the Section V concludes the paper. 


\section{Kinematic model of the robot}

Identical robots are considered such that each of them has a set of sensors and effectors (actuators) allowing it to move. The proposed controller architecture will be tested on the pioneer P3-DX robots. The P3-DX robot has a circular platform and is equipped with a locomotion device consisting of two independent driving wheels, one caster-wheel, and 16 sonar sensors to cover a field perception of $360^{\circ}$. This platform is fairly standard and offers multiple application domains; also it is easy to control.

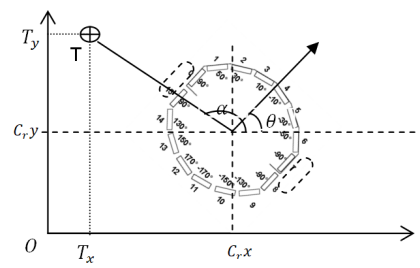

Fig. 1: Relative robot and target positions

According to the chosen platform, the robots are considered as points in the configuration space with the absolute origin $O$ in the environment. Each robot $r$ is located by its centre point denoted by $C_{r}$. The different configurations of the robot in the environment $E$ at any time are determined by the following kinematic equations:

$$
\left\{\begin{array}{l}
C_{r} x(t)=C_{r} x(t-1)+\left|V_{r}(t)\right| \cos \theta(t) \\
C_{r} y(t)=C_{r} y(t-1)+\left|V_{r}(t)\right| \sin \theta(t)
\end{array}\right.
$$

with

$$
\begin{gathered}
\left\{\begin{array}{l}
\theta(t)=\theta(t-1)+\Delta \theta(t) \\
\left|V_{r}(t)\right|=\left|V_{r}(t-1)\right|+\Delta\left|V_{r}(t)\right|
\end{array}\right. \\
\gamma(t)=\alpha(t)-\theta(t) \\
\alpha(t)=\arctan \left(\frac{T_{y}-C_{r} y}{T_{x}-C_{r} x}\right)
\end{gathered}
$$

$\alpha(t)$ is the angle of the target, $\theta(t)$ is the robot heading angle in the configuration space and $\left|V_{r}(t)\right|$ is the translation velocity of robot $r$ at time $t$. The relative angle that the robot makes with the target $T$ denoted by $\gamma(t)$ is presented in figure Fig 1.

\section{Fuzzy controller}

To correctly implement the behaviours, a series of fuzzy decision rules are used with non-conflicting checking in their realizations, because it is possible that two opposing behaviours can be triggered at the same time. For example, turn right to target tracking and turn left to avoid the obstacle [9]. To solve this problem, we introduce the notion of context. This latter allows the determination of the behaviour should be followed by the robot based on a given environmental situation. All behaviours are not applicable in the same way. For example, the behaviour target tracking will be more appropriate when the path is free. We propose describing the context through terms in fuzzy logic [13]. Specifically, we formulate the knowledge of robots about its environment as fuzzy variables with linguistic membership functions. 
The variables $\gamma(t)$ and sonar sensor data can be used as inputs variables of the fuzzy controller to effectively estimate the value of the desired heading angle $\theta(t)$ of the robot and the desired translation velocity $\left|V_{r}(t)\right|$. The value output variable $\theta(t)$ allows to robot the navigation in the environment with avoiding obstacles and at the same time the access to the target. The value of $\theta(t)$ and $\left|V_{r}(t)\right|$ is obtained by means of the Fuzzy Inference System (FIS) where we define two contexts: the first is Target Tracking and the second is Obstacle Avoidance.

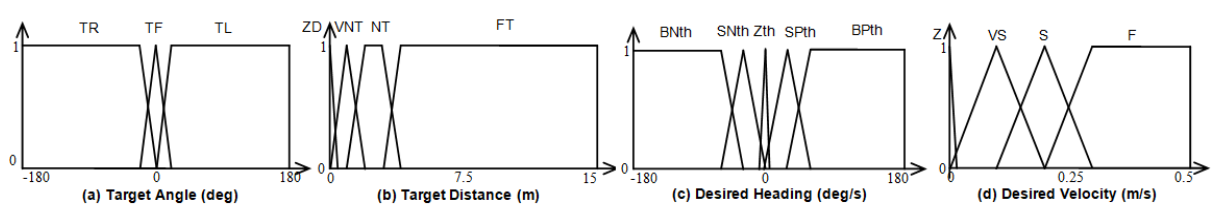

Fig. 2: Input/Output Membership Functions

Table 1: List of fuzzy rules used for implementation of target tracking behaviour.

\begin{tabular}{|c|c|c|c|c|c|c|c|}
\hline \multirow{2}{*}{$T \_D$} & \multicolumn{3}{|c|}{ Target Angle $T A$} & \multirow{2}{*}{$T \_D$} & \multicolumn{3}{|c|}{ Target Angle $T A A$} \\
\hline & TL & $\mathrm{TF}$ & TR & & TL & TF & TR \\
\hline \multirow{2}{*}{ Z } & $D_{-} \theta$ is $Z \theta$ & $D_{-} \theta$ is $Z \theta$ & $D_{-} \theta$ is $Z \theta$ & \multirow{2}{*}{ NT } & $D_{-} \theta$ is $B P \theta$ & $D_{-} \theta$ is $Z \theta$ & $D_{-} \theta$ is $B N \theta$ \\
\hline & $D_{\_} V$ is $Z$ & $D_{\_} V$ is $Z$ & $D_{-} V$ is $Z$ & & $D_{-} V$ is $S$ & $D_{-} V$ is $S$ & $D_{-} V$ is $S$ \\
\hline \multirow{2}{*}{ VNT } & $D_{-} \theta$ is $S P \theta$ & $D_{-} \theta$ is $Z \theta$ & $D_{-} \theta$ is $S N \theta$ & \multirow{2}{*}{ FT } & $D_{-} \theta$ is $B P \theta$ & $D_{-} \theta$ is $Z \theta$ & $D_{-} \theta$ is $B N \theta$ \\
\hline & $D_{-} V$ is $V S$ & $D_{-} V$ is $V S$ & $D_{-} V$ is $V S$ & & $D_{-} F$ is $S$ & $D_{-} V$ is $F$ & $D_{\_} V$ is $F$ \\
\hline
\end{tabular}

\subsection{Target tracking behaviour}

The first step, called fuzzification, consists to transform the real values of the input variables into fuzzy variables. For this, the controller uses the following universe of discourse: T_A Target Angle: \{ TL: 'Target on the Left', TF: 'Target on front', TR: 'Target on the right' $\}$, and $T D$ Target Distance: $\{$ ZD:' Zero Distance', VNT: ' Very Near Target' , 'NT: ' Near Target', FT: ' Far Target' \}.

The two output variables are the desired heading angle and the desired translation velocity : $D_{-} \theta$ Desired $\theta:\{Z \theta:$ ' Zero $\theta$ ', $B N \theta$ : ' Big Negative $\theta$ ', $S N \theta$ :' Small Negative $\theta$ ', $B P \theta$ : ' Big Positive $\theta$ ', $S P \theta$ : ' Small Positive $\theta$ '\}, abd D_V Desired velocity : $\left\{Z:^{\prime}\right.$ Zero $^{\prime}, V S$ :' VerySlow $^{\prime}, S:^{\prime}$ Slow $^{\prime}, F:^{\prime}$ Fast $\left.^{\prime}\right\}$.

The membership functions of the input variables are shown in figures Fig 2-(a); Fig 2-(b) and output variables are shown in figures Fig 2-(c); Fig 2-(d). The proposed model uses fuzzy inference system proposed in [11]. The linguistic variable value of the desired $\theta$ and the velocity is determinate using 24 fuzzy rules proposed in table 1 .

\subsection{Obstacle avoidance behaviour}

Obstacle avoidance behaviour is triggered when one of the sensors detects an obstacle. The six sensors used by the robots have a range of 5 meters. The values transmitted by these sensors are normalized in the interval [0..1]. The fuzzy input variables and their membership functions for each sensor are shown in figure Fig 3 . The outputs value of the desired $\theta$ and the velocity is determinate using 18 fuzzy rules proposed in table 2. 


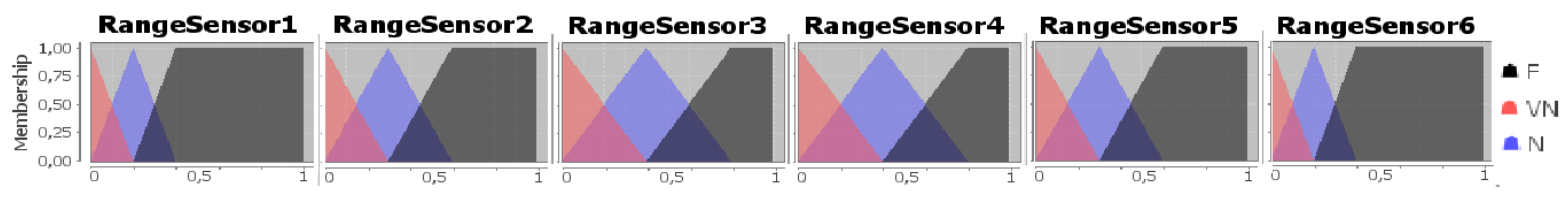

Fig. 3: Membership function of Sonar Range

Table 2: List of fuzzy rules used for implementation of obstacle avoidance behaviour.

\begin{tabular}{|c|c|c|c|c|c|c|c|}
\hline \multirow{2}{*}{ Sensor Range } & \multirow{2}{*}{$\begin{array}{l}\text { Input } \\
\text { Fuzzy Value }\end{array}$} & \multicolumn{2}{|c|}{ Output } & \multirow{2}{*}{ Sensor Range } & \multirow{2}{*}{$\begin{array}{l}\text { Input } \\
\text { Fuzzy Value }\end{array}$} & \multicolumn{2}{|c|}{ Output } \\
\hline & & $D_{-} \theta$ & $D_{-} V$ & & & $D_{-} \theta$ & $D \_V$ \\
\hline \multirow{3}{*}{ Sensor 1} & $\mathrm{VN}$ & $B N \theta$ & VS & \multirow{3}{*}{ Sensor 4} & $\mathrm{VN}$ & $B P \theta$ & $\mathrm{Z}$ \\
\hline & $\mathrm{N}$ & $S N \theta$ & VS & & $\mathrm{N}$ & $S P \theta$ & VS \\
\hline & F & $Z \theta$ & $\mathrm{S}$ & & F & $S P \theta$ & $\mathrm{F}$ \\
\hline \multirow{3}{*}{ Sensor 2} & VN & $B N \theta$ & VS & \multirow{3}{*}{ Sensor 5} & VN & $B P \theta$ & VS \\
\hline & $\mathrm{N}$ & $N \theta$ & $\mathrm{S}$ & & $\mathrm{N}$ & $P \theta$ & $\mathrm{S}$ \\
\hline & F & $\operatorname{SN\theta }$ & S & & F & $S P \theta$ & S \\
\hline \multirow{3}{*}{ Sensor3 } & VN & $B N \theta$ & $\mathrm{Z}$ & \multirow{3}{*}{ Sensor 6} & VN & $B P \theta$ & VS \\
\hline & $\mathrm{N}$ & $S N \theta$ & VS & & $\mathrm{N}$ & $S P \theta$ & VS \\
\hline & $\mathrm{F}$ & $\operatorname{SN\theta } \theta$ & $\mathrm{F}$ & & $\mathrm{F}$ & $Z \theta$ & $\mathrm{S}$ \\
\hline
\end{tabular}

\subsection{Defuzzification of output variables}

When all the rules are put, we define the method of defuzzification. This step makes it possible to transform the linguistic variable values used by the fuzzy controller from fuzzy domain to the domain of the real values. The choice of the defuzzification method is usually conditioned by a compromise between the ease of implementation and performance of calculating. In this paper, we used the centre of gravity method [9] which can be determined using the following general relationship:

$$
\theta^{* C O G}=\frac{\int \theta \psi(\theta) d_{\theta}}{\int \psi(\theta) d_{\theta}}, v^{* C O G}=\frac{\int v \psi(v) d_{v}}{\int \psi(v) d_{v}}
$$

were $\theta^{* C O G}$ is the real value of desired angle and $v^{* C O G}$ is the desired velocity obtained by the Centre of Gravity method.

\subsection{Behaviors combination}

To activate the appropriate FIS, the behaviour combination process uses a function Context $(R)$ which returns 1 if an obstacle is detected, otherwise it returns 0 ; such that $R=\left\{r_{1}, r_{2}, . ., r_{6}\right\}$ represent the values returned by the sensors $s_{1}$ respectively $\left(s_{2}, . ., s_{6}\right)$.

$$
\text { Context }(R)=\left\{\begin{array}{l}
1 \text { if }\left(\min _{\forall r_{i} \in R} r_{i}\right)<\text { RangeMax } \\
0 \text { if }\left(\min _{\forall r_{i} \in R} r_{i}\right)=\text { RangeMax }
\end{array}\right.
$$

This function is implemented such that RangeMax is the maximum range value of sensors fixed at 5 meters. The combination of behaviour is determined by the fuzzy controller according to the following Behavior Selection Algorithm.

\section{Simulation result}

In order to experimentally demonstrate the role of navigation controller, we have used a realistic simulation environment. Experimentation were performed using MobileSim Simulation Environment [12]. The experiments were conducted by 


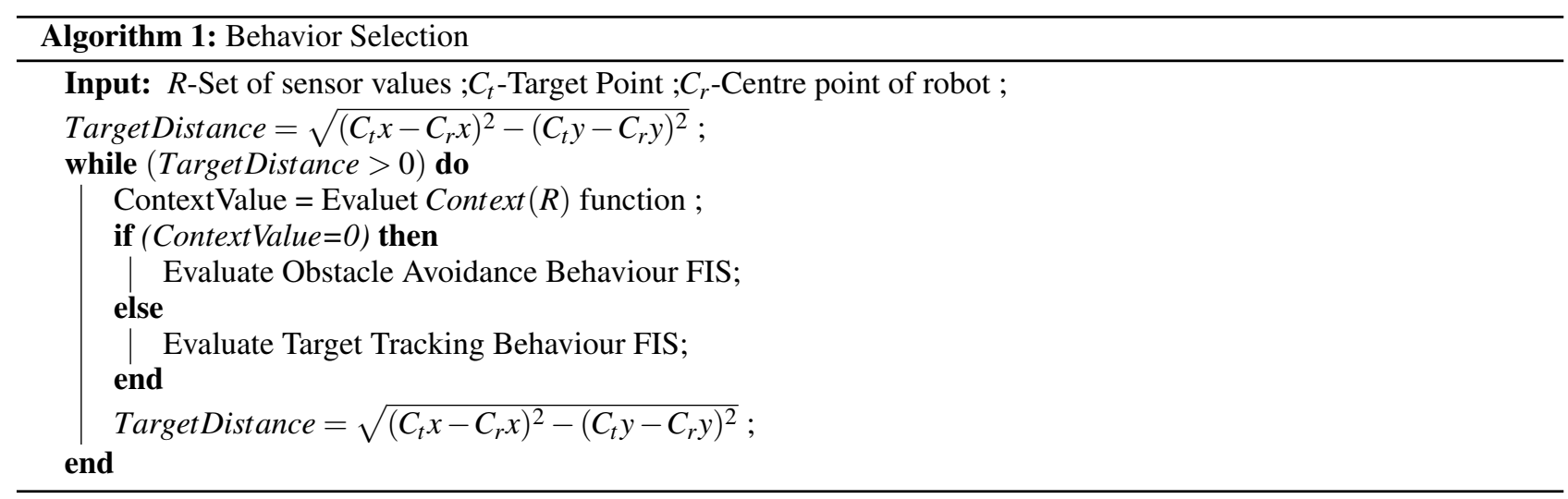

using a team of Pioneer P3DX robots in an enclosed space with dimension 40 x 25 meters included fixed obstacles. The figure Fig 4 shows the environment that we have used to test the proposed architecture. We have realized several simulations with different configurations. In figure Fig 4.(a) we have simulated a robot that has broken down, the traces of movement of the active robot show that the latter considered the failed robot as a fixed obstacle. In figure Fig 4.(b) we have realized the same simulation, but we have turned on two robots simultaneously. The traces show that the two robots have not been intersected and the figures Fig 4.(c) show that the robot paths cross in the two points A and B. The mechanism of selection behaviours makes it possible to avoid the collisions. Finally, the figure Fig 4.(d) clearly shows that robots always arrive at the target without collision with any obstacles or with another robot.

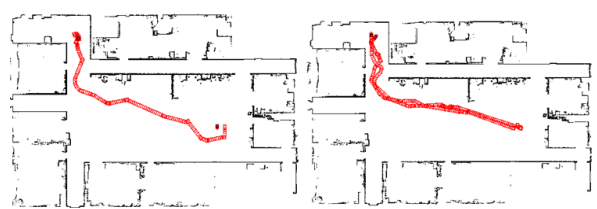

(a) (b)

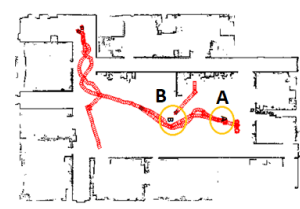

(c)

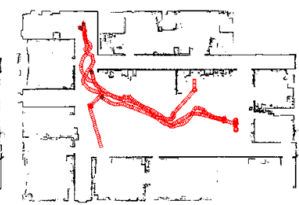

(d)

Fig. 4: Paths generated by combination behaviours process in navigation multi robots system

\section{Conclusion}

Fuzzy logic can be a useful tool for the navigation process in the distributed MRS and more particularly in a dynamic and uncertain environment. It can be used to formulate the different information received and observed by robots in the form of linguistic terms similar to those used by human. From this point of view, we can design robots with reasoning abilities which are similar to those found in humans. This may be possible if we choose a set of rules that allow the use these linguistic variables to conclude other information in order to make a particular decision. For this, it is necessary to use an appropriate architecture, especially when the functionalities of the robots are effectively separated. The process of selection behaviours proposed shows a great flexibility to implement the different navigation approaches in multi-robot systems.

\section{Competing interests}

The authors declare that they have no competing interests. 


\section{Authors' contributions}

All authors have contributed to all parts of the article. All authors read and approved the final manuscript.

\section{References}

[1] Buffet, O., Dutech, A., Charpillet, F: Adaptive combination of behaviors in an agent. In: 9th European Conference on Artificial Intelligence, pp. 48-52. (2010)

[2] Liu, C., Kroll, A. : A centralized multi-robot task allocation for industrial plant inspection by using a* and genetic algorithms. In: International Conference on Artificial Intelligence and Soft Computing, pp. 466-474. Springer, Berlin, Heidelberg (2012)

[3] Liu, F., Liang, S., Xian, X: Multi-robot task allocation based on utility and distributed computing and centralized determination. In: The 27th Chinese Control and Decision Conference (2015 CCDC), pp. 3259-3264. IEEE, (2015)

[4] Chi, K. H., Lee, M. F. R.: Obstacle avoidance in mobile robot using neural network. In: Int. Conf. on Consumer Electronics, Communications and Networks (CECNet), pp. 5082-5085. IEEE, (2011)

[5] Hoang, T. T., Hiep, D. T., Duong, P. M., Van, N. T. T., Duong, B. G., Vinh, T. Q.: Proposal of algorithms for navigation and obstacles avoidance of autonomous mobile robot. In: IEEE 8th Conference on Industrial Electronics and Applications (ICIEA), pp. 1308-1313, IEEE, (2013)

[6] Aouf, A., Boussaid, L., Sakly, A.: Same fuzzy logic controller for two-wheeled mobile robot navigation in strange environments. Journal of Robotics 2019, (2019)

[7] Cheam, S. R., Saman, A. B. S. : Developing algorithm for object tracking using passive sensors. In:5th International Conference on Intelligent and Advanced Systems (ICIAS), pp. 1-5, IEEE, (2014)

[8] Arrichiello, F., Chiaverini, S., Mehta, V. K.: Experiments of obstacles and collision avoidance with a distributed multi-robot system. In:IEEE International Conference on Information and Automation, pp. 727-732, IEEE, (2012)

[9] Apandi, N. I. A., Martin, A. : The Integration of Fuzzy Logic System for Obstacle Avoidance Behavior of Mobile Robot. International Journal of Electrical Engineering and Applied Sciences (IJEEAS) 2(1), 31-38 (2019)

[10] Zadeh, L. A.: Fuzzy sets. Information and control 8(3), 338-353 (1965)

[11] Mamdani, E. H., Assilian, S.: An experiment in linguistic synthesis with a fuzzy logic controller. International journal of manmachine studies 7(1), 1-13 (1975)

[12] Omron ('MobileSim'), http://robots.mobilerobots.com/wiki/MobileSim.. Last accessed 17 Jan 2019. 